Research Paper

\title{
The prognostic value of Cyclin-Dependent Kinase 5 and Protein Phosphatase 2A in Gastric Cancer
}

\author{
Jian-Xian Lin ${ }^{1,2,3^{*}}$, Xin-Sheng Xie ${ }^{1,2,3^{*}}$, Xiong-Feng Weng1,2,3, Chao-Hui Zheng1,2,3, Jian-Wei Xie ${ }^{1,2,3}$, Jia-Bin \\ Wang1,2,3, Jun Lu1,2,3, Qi-Yue Chen ${ }^{1,2}$, Long-Long Cao ${ }^{1,2}$, Mi Lin ${ }^{1,2}$, Ru-Hong Tu ${ }^{1,2}$, Chang-Ming Huang ${ }^{1,2,3}$, \\ Ping $\mathrm{Li}^{1,2,3 \bowtie}$ \\ 1. Department of Gastric Surgery, Fujian Medical University Union Hospital, Fuzhou, Fujian Province, China \\ 2. Key Laboratory of Ministry of Education of Gastrointestinal Cancer, Fujian Medical University, Fuzhou, Fujian Province, China \\ 3. Fujian Key Laboratory of Tumor Microbiology, Fujian Medical University, Fuzhou, Fujian Province, China \\ *Jian-Xian Lin and Xin-Sheng Xie contributed equally to this work and should be considered co-first authors. \\ $\square$ Corresponding authors: Ping Li, E-mail:Pingli811002@163.com; Chang-Ming Huang, E-mail: hcmlr2002@163.com; Department of Gastric Surgery, Fujian \\ Medical University Union Hospital, No.29 Xinquan Road, Fuzhou 350001, Fujian Province, China. Telephone: +86-591-83363366; Fax: +86-591-83363366 \\ (C) Ivyspring International Publisher. This is an open access article distributed under the terms of the Creative Commons Attribution (CC BY-NC) license \\ (https://creativecommons.org/licenses/by-nc/4.0/). See http://ivyspring.com/terms for full terms and conditions.
}

Received: 2018.05.02; Accepted: 2018.08.02; Published: 2018.10.22

\begin{abstract}
Purpose To discuss the relationship between the clinicopathological data, long-term survival of gastric cancer patients and different expression levels of Cyclin-Dependent Kinase 5 (CDK5) and Protein Phosphatase 2A (PP2A).

Method The expression levels of CDK5 and PP2A were detected by immunohistochemistry in specimens from 124 patients with primary gastric cancer. The correlation among the expression of CDK5 and PP2A, clinicopathological factors and prognosis was investigated.

Result The expression level of CDK5 was correlated with the TNM stage $(p=0.030)$ and $N$ stage $(p=0.001)$, while the expression level of PP2A was correlated with the TNM stage and N stage $(p=0.001$ and $p=0.004)$ as well as the degree of differentiation $(p=0.046)$. The expression of CDK5 was positively correlated with the expression of PP2A in gastric cancer. Co-expression of CDK5 and PP2A is an independent prognostic factor that affected overall survival, and provided more accurate prognostic value for the overall survival of gastric cancer patients.

Conclusion The expression of CDK5 and PP2A is positively correlated in gastric cancer. Co-expression of CDK5 and PP2A was an independent prognostic factor in patients with gastric cancer.
\end{abstract}

Key words: CDK5; PP2A; prognosis; gastric cancer

\section{Introduction}

Gastric cancer (GC) is the fifth most common malignant cancer worldwide and is the third most common cause of cancer-related death [1]. China has a high incidence of gastric cancer, as more than 400,000 new cases occur every year, which account for $42 \%$ of the world's gastric cancer cases [2]. Considering that the symptoms of early gastric cancer are not highly specific, misdiagnosis and missed diagnosis may occur. Most patients are in the middle and late stages of disease at the time of their first visit to a healthcare professional, which seriously affects the treatment strategy and prognosis. This is also one of the major causes of the high overall mortality of gastric cancer. Therefore, it is of great importance to find new molecular markers that can be used to evaluate the prognosis and to develop novel therapies for gastric cancer.

CDK5 is an important serine/threonine kinase 
that is expressed in many organisms. CDK5 is widely expressed in the nervous system and other tissues, and is involved in a variety of pathophysiological processes $[3,4]$. In recent years, it has been suggested that $\mathrm{CDK} 5$ is involved in the occurrence and development of many types of malignant tumors [5]. It has been reported that activation of CDK5 can phosphorylate the $\mathrm{Rb}$ protein, release E2F1 into the nucleus, and thus activate the expression of downstream factors and promote the development of medullary thyroid carcinoma [6]. The inhibition of CDK5 activity can interfere with the Notch pathway, inhibit the activation of the Notch intracellular domain (NICD), and thus inhibit tumor growth [7]. In addition, CDK5 plays an important role in the proliferation, invasion and metastasis of malignant tumors such as liver cancer and prostate cancer among others [8,9]. However, in previous studies, we found that low expression of CDK5 was associated with the overall poor survival of gastric cancer patients. The nuclear accumulation of CDK5 inhibited the proliferation and tumorigenicity of human gastric cancer cells, which suggests that CDK5 might play a role as a tumor suppressor in the carcinogenesis and development of gastric cancer [10].

PP2A is a widespread Ser/Thr protein phosphatase in eukaryotes. The structural subunit (PR65/ A: 65 $\mathrm{kDa}$ ), the regulatory subunit (B subunit: 50 130 kDa) and the catalytic subunit (PP2Ac: $36 \sim 38 \mathrm{kDa}$ ) form a trimer; this complex, is involved in the cell cycle, DNA replication, signal transduction, cell differentiation, malignant transformation and other cellular and biological events. PP2A was traditionally considered to be related to the occurrence and development of neurodegenerative diseases, tumors and other diseases [11-13]. Aberrant PP2A expression is associated with the occurrence and development of many malignant tumors, such as colorectal cancer, breast cancer, lung cancer and glioma [14-17]. Activation of PP2A can promote apoptosis, dedifferentiation and can inhibit the proliferation of tumor cells, and thus activation of PP2A is a potential target for the treatment of malignant tumors [18, 19]. Louis et al [20] Found that a knockout of the PP2A subunit (Ppp2r5d) in mice could affect the activities of CDK5 and GSK-3 $\beta$, decrease the activity of CDK5 phosphatase and increase the activity of GSK-3 $\beta$, which lead the to the development of space restricted tau disease in these knockout mice. However, the expression of CDK5 and PP2A in gastric cancer and its influence on prognosis have not been reported. Therefore, the expression of CDK5 and PP2A was detected in 124 cases of gastric cancer after radical gastrectomy. The correlation among CDK5 and PP2A expression, the clinicopathological features and prognosis was analyzed.

\section{Materials and Methods Human gastric tumor tissues}

The gastric cancer specimens used in this study were collected from January 2013 to December 2014 in the Department of Gastric Surgery, Union Hospital of Fujian Medical University, from patients who underwent D2 lymph node dissection and gastrectomy for gastric cancer. Cases with incomplete clinical data were excluded, which resulted in 124 cases. All patients received their first diagnosis of gastric cancer and received no other treatment, such as neoadjuvant chemotherapy or chemoradiotherapy, before surgery. All diagnoses were confirmed by pathology after surgery, and each case had detailed clinicopathological data. Gastric cancer was confirmed by hematoxylin and eosin (H\&E) staining in all cases. The clinicopathological data of 124 patients with GC included age, sex, size of the primary tumor, location of the primary tumor, degree of differentiation, histological type (Bormann type), depth of invasion, lymph node metastasis, vascular invasion and TNM stage (according to the TNM classification of gastric cancer (eighth edition) of the International Union against Cancer (2016)); the pathological stage of the tumor was reassessed. This study was approved by the ethics committee of Fujian Medical University Union Hospital and written consent was obtained from all patients.

\section{Immunohistochemistry (IHC)}

Paraffin sections containing sufficient formalin fixed tumor tissue were sectioned continuously at a thickness of $4 \mu \mathrm{m}$ and were mounted on silage coated slides for immunohistochemical analysis. The slices were deparaffinized with xylene and rehydrated in $95 \%, 85 \%$ and $75 \%$ ethanol. Antigen retrieval was performed by subjecting the slides to high-pressure sterilization at $121^{\circ} \mathrm{C}$ for $2 \mathrm{~min}$ in $0.01 \mathrm{~mol} / \mathrm{L}$ sodium citrate buffer solution ( $\mathrm{pH}$ 6.0). Endogenous peroxidase activity was blocked by incubating the slides with $3 \% \mathrm{H} 2 \mathrm{O} 2$ at room temperature for $10 \mathrm{~min}$. The slices were then washed in phosphate buffered saline (PBS) solution and blocked in 10\% goat serum (Zhongshan Biotechnology Co, Ltd.) for 30 minutes. Next, the sections were incubated with diluted rabbit anti-human CDK5 (ab24189 diluted 1: 200.USA) or rabbit anti-human PP2Aa (ab32104n diluted 1: 150; Abcam, Cambridge, MA, USA) overnight in a humidified chamber at $4^{\circ} \mathrm{C}$. After three washes in PBS, the sections were incubated with the secondary antibody conjugated to horseradish peroxidase at room temperature for 30 minutes. The signal was developed with diaminobenzidine solution, which 
was followed by counterstaining in $20 \%$ hematoxylin. Finally, all slides were dehydrated and mounted on cover glass. For negative controls, antibody diluent was substituted for the primary antibody.

\section{Evaluation of Immunohistochemistry Intensity}

The IHC of tissue sections was examined by two experienced pathologists, who scored the slides according to the intensity of cell staining and the proportion of positively stained tumor cells. In the event of a discrepancy, the sample was reassessed, and a consensual scoring criterion was selected for the evaluation. CDK5 is primarily expressed in the cytoplasm and/or the nucleus of cancer cells, and the immunohistochemical staining is yellow to brown. The expression pattern of the protein in the tumor was one in which the cells were either all positive or completely negative. Therefore, the proportion of tumor cells that were stained is not available. The standard for the evaluation of CDK5 staining intensity was as follows: (a1): no staining (score of 0), (a2): weak staining (score of 1), (a3): moderate staining (score of 2) and (a4) strong staining (score of 3) (Figure $1 \mathrm{~A})$. For CDK5, a score $\leq 1$, is considered low expression, while all other scores indicate high expression. Unlike CDK5, the expression pattern of
PP2A is not all or none, and thus the standard for the evaluation of PP2A staining intensity is the same as that of CDK5. The positive proportion of stained tumor cells was scored as follows: (b5): $\leq 5 \%$ positive cells (score of 0 ), (b6): $6 \%$ to $25 \%$ positive cells (score of 1 ), (b7): $26 \%$ to $50 \%$ positive cells (score of 2 ), (b8): $\geq$ $51 \%$ positive cells (score of 3 ) (Figure 1B). If the total score (percentage score $x$ intensity score) was less than 3, PP2A protein expression was considered low, but if the score was 4 or higher, PP2A protein expression was considered high.

\section{Follow-up}

All patients were followed up once every 3 months for the first 2 years and were then, followed up every six months for the next 3-5 years. The last follow-up time point was December 2017. Follow-up routine examinations, including a physical examination, laboratory tests (CA19-9, CEA and CA72-4), chest X-ray, abdominal CT, B Ultrasound, and gastroscopy were performed each year. The total survival time is defined as the time from surgery to the last follow-up, or the time of death, or the expiration of the follow-up database (e.g., lost to follow-up, death from other diseases, etc.)
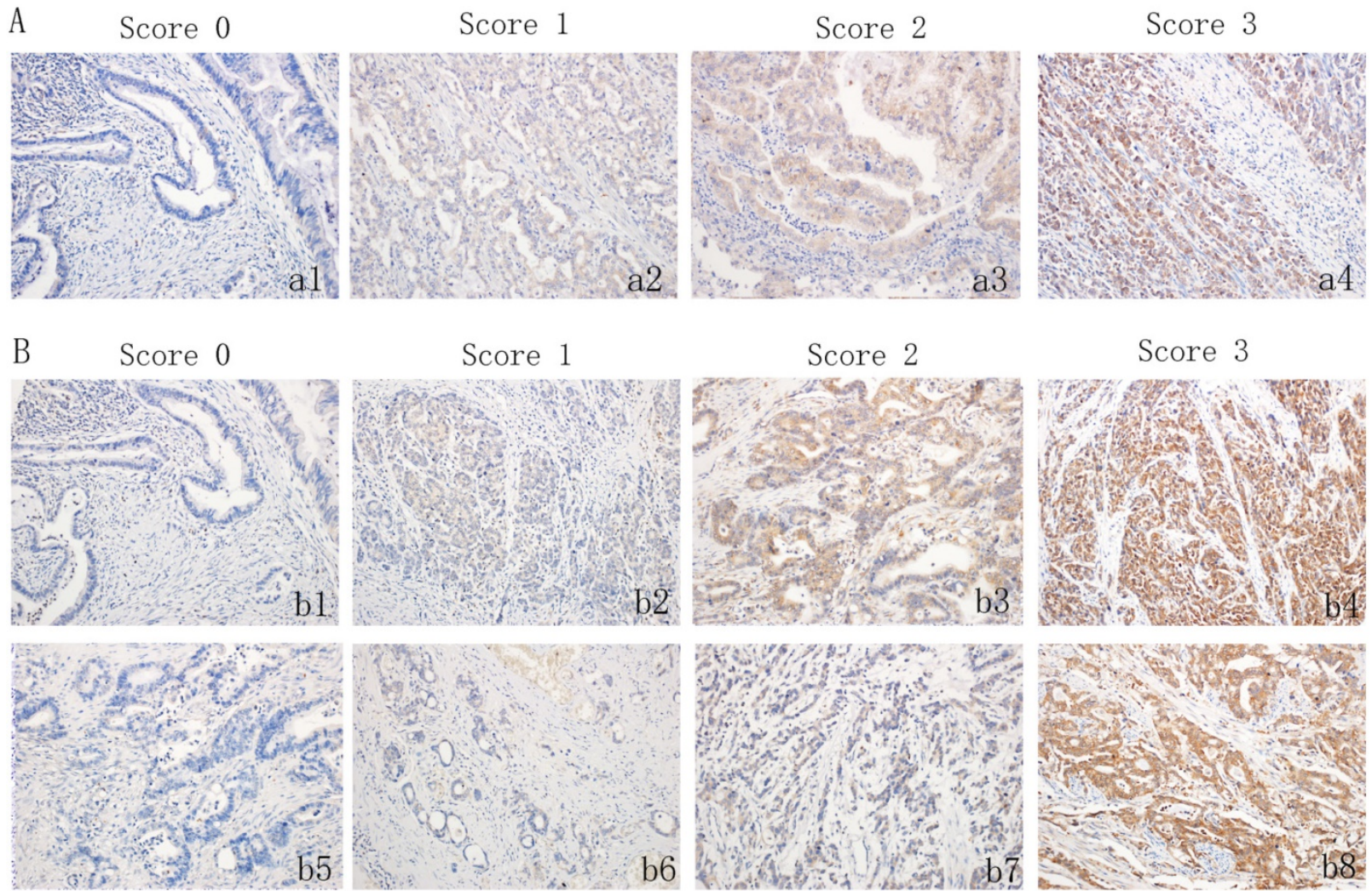

Figure 1. Immunohistochemistry staining for CDK5 and PP2A expression in gastric cancerous tissue and the criteria for immunohistochemistry scoring. (A) Intensity scores for CDK5 expression in gastric cancer tissue. (a1): no staining (score 0), (a2): weak staining (score 1), (a3): moderate staining (score 2) and (a4) strong staining (score 3). (B) Distribution score and intensity score for PP2A expression in gastric cancer tissue. (b1): no staining (score 0), (b2): weak staining (score 1), (b3): moderate staining (score 2 ) and (b4) strong staining (score 3 ). (b5): $\leq 5 \%$ positive cells (score 0 ), (b6): $6 \%$ to $25 \%$ positive cells (score 1), (b7): $26 \%$ to $50 \%$ positive cells (score 2 ), (b8): $\geq 51 \%$ positive cells (score 3 ). 

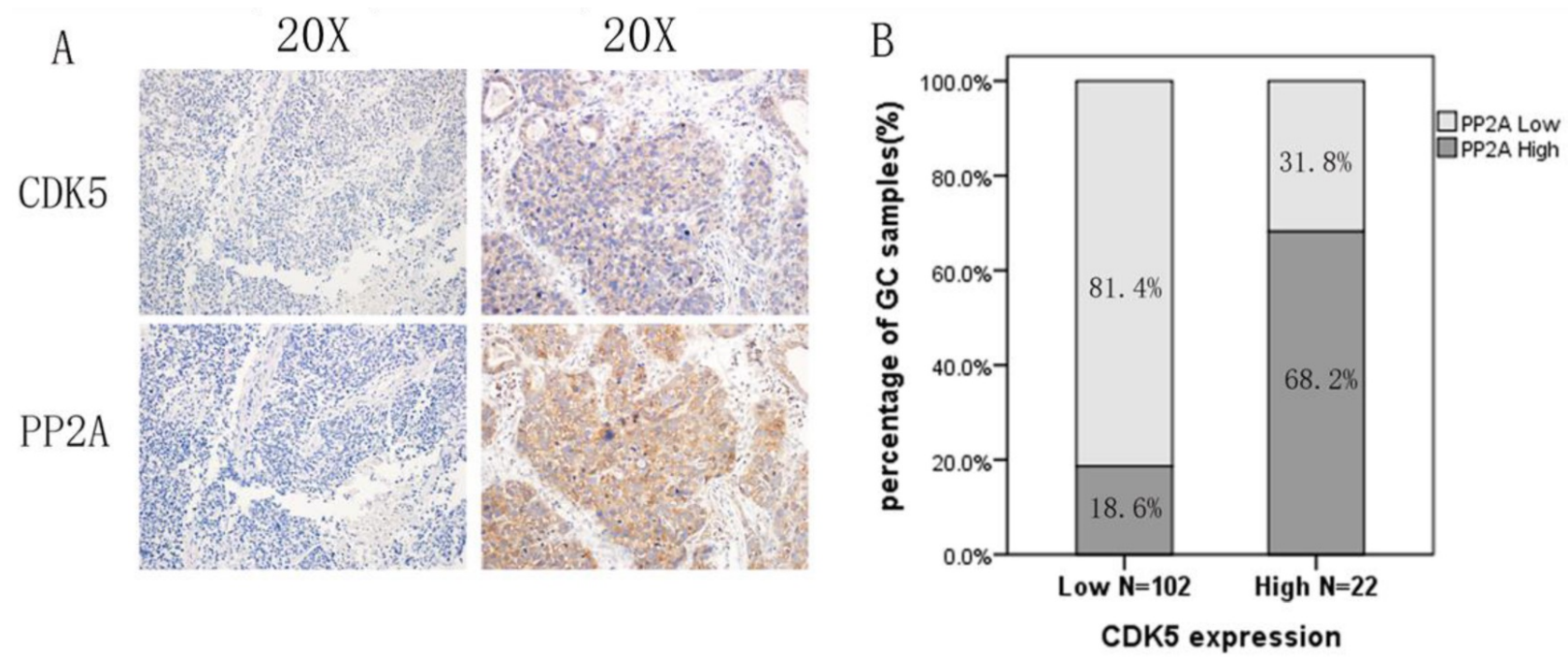

Figure 2. CDK5 is positive correlated with PP2A expression in gastric cancer. A: High CDK5 and PP2A expression in the same patient. B: The positive expression rate of PP2A was $68.2 \%$ in gastric cancer tissues with high expression of CDK5, and negative expression rate of PP2A was $81.4 \%$ in stomach tissues with low expression of CDK5.

\section{Statistical analysis}

All the data were processed by SPSS23.0 statistical software package. Appropriate test methods, such as the $\chi 2$ test or Fisher's exact test, were selected according to the type of variables and the purpose of comparison. The survival rate was calculated by the Kaplan-Meier method, and the subsequent survival curve was plotted. The log-rank test was used to compare the survival rates. Cox regression was used to analyze the independent factors that affected the prognosis. The area under the ROC curve was used to compare the prognostic ability of different indexes. The difference was statistically significant when $\mathrm{p}<0.05$.

\section{Results}

\section{Expression Status of CDK5 and PP2A in Gastric Cancer}

Of the 124 patients with primary gastric cancer, 102 patients had low expression of CDK5 (82.2\%), and 22 patients had high expression (17.8\%).In addition, 90 patients had low expression of PP2A (72.6\%), and 34 patients had high expression of PP2A (27.4\%). Among the patients with low expression of CDK5, $81.4 \%$ showed low expression of PP2A. A significant positive correlation was observed between the expression levels of CDK5 and PP2A in gastric cancer tissue ( $p<0.001$, Figure 2$)$.

\section{Relationship between the expression of CDK5 and PP2A and the Clinicopathological data of patients with gastric cancer}

According to the different expression levels of CDK5 and PP2A, the correlation factor analysis showed that the expression levels of CDK5 and PP2A in gastric cancer were correlated with $\mathrm{N}$ stage (CDK5: $p=0.001$, PP2A: $p=0.004)$ and TNM (CDK5: $p=0.030$, PP2A: $p=0.001)$ stage. In addition, PP2A is also related to the differentiation level of the tumor tissue $(p=0.046$; Table 1). Furthermore, the expression levels of CDK5 and PP2A were also correlated with the TNM stage $(p=0.008)$ and $N$ stage $(p=0.006$; Table 2$)$.

\section{Prognostic value of CDK5 and PP2A expression}

The median follow-up time was 28.5 months, and the 3 -year overall survival rate was $70.6 \%$.The survival analysis showed that the 3-year overall survival rate of gastric cancer patients with low expression of CDK5 was $69.9 \%$, which was lower than that of patients with high expression of CDK5 (95.5\%). This difference was statistically significant (Figure 3 A). The prognosis of gastric cancer patients with low expression of PP2A was also worse than that of patients with high expression of PP2A $(68.0 \%$ vs. $91.2 \%$, Figure $3 \mathrm{~B}$ ). The 3-year overall survival rate of patients with low co-expression of CDK5 and PP2A was $66.5 \%$, which was significantly lower than that of patients with high expression of CDK5 and/or PP2A (90.2\%, Figure 4 A). Further analysis showed that the prognosis of patients with high expression of CDK5 and PP2A was better than that of patients with high expression of CDK5 or PP2A, and that patient with low expression of CDK5 and PP2A exhibited the worst prognosis, (Figure 4 B).

\section{Univariate and multivariate analyses of prognosis in the entire group}

A univariate analysis showed that the 3-year 
overall survival rate was correlated with $\mathrm{T}$ stage $(p=0.033), N$ stage $(p=0.043)$, TNM stage $(p<0.001)$, and the expression of CDK5 $(p=0.015)$ and PP2A $(p=0.008)$ in tumor tissues. The co-expression of CDK5 and PP2A in tumor tissues $(\mathrm{p}=0.004)$ was also demonstrated (Table 3). A multivariate analysis showed that the TNM stage $(p=0.037)$ and the co-expression of CDK5 and PP2A $(p=0.048)$ were independent prognostic factors in patients with gastric cancer (Table 4).

Table 1. Relationships between CDK5 and PP2A protein expressions in gastric cancer tissues and various clinicopathological variables

\begin{tabular}{|c|c|c|c|c|c|c|c|c|c|}
\hline \multirow[t]{2}{*}{ Variables } & \multirow[t]{2}{*}{ Total } & \multicolumn{4}{|c|}{ CDK5 expression } & \multicolumn{4}{|c|}{ PP2A expression } \\
\hline & & Low & high & $\mathrm{X}^{2}$ & $\mathbf{P}$ & low & high & $\mathbf{X}^{2}$ & $\mathbf{P}$ \\
\hline Gender & & & & 0.203 & 0.653 & & & 0.468 & 0.494 \\
\hline Male & 97 & 79 & 18 & & & 69 & 28 & & \\
\hline Female & 27 & 23 & 4 & & & 21 & 6 & & \\
\hline Age (years) & & & & 2.184 & 0.139 & & & 0.018 & 0.894 \\
\hline$>60$ & 85 & 67 & 18 & & & 62 & 23 & & \\
\hline$\leq 60$ & 39 & 35 & 4 & & & 28 & 11 & & \\
\hline Tumor size $(\mathrm{cm})$ & & & & 1.284 & 0.257 & & & 0.213 & 0.644 \\
\hline$>5$ & 47 & 41 & 6 & & & 33 & 14 & & \\
\hline$\leq 5$ & 77 & 61 & 16 & & & 57 & 20 & & \\
\hline Tumor location & & & & 1.085 & 0.781 & & & 0.371 & 0.946 \\
\hline Lower $1 / 3$ & 53 & 44 & 9 & & & 38 & 15 & & \\
\hline Middle $1 / 3$ & 18 & 16 & 2 & & & 14 & 4 & & \\
\hline Upper $1 / 3$ & 41 & 33 & 8 & & & 29 & 12 & & \\
\hline More than $1 / 3$ & 22 & 19 & 3 & & & 9 & 3 & & \\
\hline Bormann type & & & & 0.109 & 0.741 & & & 2.052 & 0.152 \\
\hline I+II type & 25 & 20 & 5 & & & 21 & 4 & & \\
\hline III+IV type & 99 & 82 & 17 & & & 69 & 30 & & \\
\hline \multicolumn{3}{|c|}{ Degree of differentiation } & & 2.827 & 0.093 & & & 3.999 & 0.046 \\
\hline Well/moderate & 48 & 36 & 12 & & & 30 & 18 & & \\
\hline Poor and not & 76 & 66 & 10 & & & 60 & 16 & & \\
\hline \multicolumn{2}{|l|}{ Histological type } & & & 0.948 & 0.814 & & & 7.384 & 0.061 \\
\hline Papillary & 58 & 48 & 10 & & & 44 & 14 & & \\
\hline Tubular & 36 & 28 & 8 & & & 22 & 14 & & \\
\hline Mucinous & 9 & 8 & 1 & & & 5 & 4 & & \\
\hline Signet-ring cell & 21 & 18 & 3 & & & 19 & 2 & & \\
\hline \multicolumn{2}{|c|}{ Depth of invasion } & & & 2.944 & 0.086 & & & 0.195 & 0.659 \\
\hline $\mathrm{T} 1+\mathrm{T} 2$ & 19 & 13 & 6 & & & 13 & 6 & & \\
\hline $\mathrm{T} 3+\mathrm{T} 4$ & 105 & 89 & 16 & & & 77 & 28 & & \\
\hline \multicolumn{3}{|c|}{ Lymph node metastasis } & & 10.288 & 0.001 & & & 8.376 & 0.004 \\
\hline Negative & 18 & 10 & 8 & & & 8 & 10 & & \\
\hline Positive & 106 & 92 & 14 & & & 82 & 24 & & \\
\hline TNM stage & & & & 4.714 & 0.030 & & & 10.957 & 0.001 \\
\hline I+II & 38 & 27 & 11 & & & 20 & 18 & & \\
\hline III+IV & 86 & 75 & 11 & & & 70 & 16 & & \\
\hline Vessel invasion & & & & 0.167 & 0.683 & & & 0.334 & 0.563 \\
\hline Negative & 78 & 65 & 13 & & & 58 & 20 & & \\
\hline Positive & 46 & 37 & 9 & & & 32 & 14 & & \\
\hline
\end{tabular}

\section{The relationship between the expression of CDK5 and PP2A and the duration of TNM stage}

As shown in Figure 5, when CDK5 or PP2A expression is low, the TNM stage of gastric cancer tends to be advanced, which indicates that they are negatively correlated. We established an ROC curve to compare the expression of CDK5 alone, the expression of CDK5 and PP2A together with TNM stage in the prediction of gastric cancer prognosis. The results showed that the area under the curve of the combination of CDK5 and PP2A (AUC: $0.639,95 \% \mathrm{CI}$ : 0.534-0.743, $\mathrm{p}=0.020)$ was larger than that of $\mathrm{CDK} 5$ or PP2A expression alone (CDK5: AUC: 0.599, 95\%CI: 0.493-0.704, $p=0.098$. PP2A: AUC: $0.622,95 \% \mathrm{CI}$ : $0.517-0.726, \mathrm{p}=0.041)$. In addition, the prognostic value of the combined expression of CDK5 and PP2A is closer to that of the TNM stage (AUC: $0.685,95 \% \mathrm{CI}$ : 0.591-0.780, Figure 6).

Table 2. Relationships between different CDK5 and PP2A protein expression status in gastric cancer tissues and various clinicopathological variables

\begin{tabular}{|c|c|c|c|c|c|}
\hline Variables & Total & $\begin{array}{l}\text { CDK5 and } \\
\text { PP2A low }\end{array}$ & $\begin{array}{l}\text { CDK5 and/ or } \\
\text { PP2A high }\end{array}$ & $x^{2}$ & $\mathbf{P}$ \\
\hline \multicolumn{6}{|l|}{ Gender } \\
\hline Male & 97 & 62 & 35 & 1.833 & 0.176 \\
\hline Female & 27 & 21 & 6 & & \\
\hline \multicolumn{6}{|l|}{ Age (years) } \\
\hline$>60$ & 85 & 56 & 29 & 0.135 & 0.713 \\
\hline$\leq 60$ & 39 & 27 & 12 & & \\
\hline \multicolumn{6}{|l|}{ Tumor size $(\mathrm{cm})$} \\
\hline$>5$ & 46 & 30 & 16 & 0.215 & 0.643 \\
\hline$\leq 5$ & 77 & 47 & 30 & & \\
\hline \multicolumn{6}{|l|}{ Tumor location } \\
\hline Lower $1 / 3$ & 53 & 34 & 19 & 0.787 & 0.853 \\
\hline Middle 1/3 & 18 & 13 & 5 & & \\
\hline Upper $1 / 3$ & 41 & 27 & 14 & & \\
\hline More than $1 / 3$ & 12 & 9 & 3 & & \\
\hline \multicolumn{6}{|l|}{ Bormann type } \\
\hline I+II type & 25 & 19 & 6 & 1.163 & 0.281 \\
\hline III+IV type & 99 & 64 & 35 & & \\
\hline \multicolumn{6}{|c|}{ Degree of differentiation } \\
\hline Well/moderate & 48 & 28 & 20 & 2.618 & 0.106 \\
\hline Poor and not & 76 & 55 & 21 & & \\
\hline \multicolumn{6}{|l|}{ Histological type } \\
\hline Papillary & 58 & 40 & 18 & 1.999 & 0.573 \\
\hline Tubular & 36 & 22 & 14 & & \\
\hline Mucinous & 9 & 5 & 4 & & \\
\hline Signet-ring cell & 21 & 16 & 5 & & \\
\hline \multicolumn{6}{|c|}{ Depth of invasion } \\
\hline $\mathrm{T} 1+\mathrm{T} 2$ & 19 & 12 & 7 & 0.145 & 0.704 \\
\hline $\mathrm{T} 3+\mathrm{T} 4$ & 104 & 71 & 34 & & \\
\hline \multicolumn{6}{|c|}{ Lymph node metastasis } \\
\hline Negative & 18 & 7 & 11 & 7.484 & 0.006 \\
\hline Positive & 106 & 76 & 30 & & \\
\hline \multicolumn{6}{|l|}{ TNM stage } \\
\hline $\mathrm{I}+\mathrm{II}$ & 38 & 19 & 19 & 7.100 & 0.008 \\
\hline III+IV & 86 & 64 & 22 & & \\
\hline \multicolumn{6}{|l|}{ Vessel invasion } \\
\hline Negative & 78 & 53 & 25 & 0.098 & 0.755 \\
\hline Positive & 46 & 30 & 16 & & \\
\hline
\end{tabular}

\section{Discussion}

In recent years, the diagnosis and treatment of gastric cancer have improved, but this cancer is still one of the most lethal malignant tumors. The exploration of specific biomarkers and effective therapeutic targets for gastric cancer is of great significance to 
promote the early diagnosis of gastric cancer and to improve the efficacy of molecularly targeted drugs. It has been reported that CDK5 can bind to its target protein and modify that protein so that it has an important biological function. The function of CDK5 is closely related to that of other binding proteins such as p35, p39, p27 and crm-1, among others [21-23]. Recently, we used co-immunoprecipitation and mass spectrometry to search for binding proteins and potential downstream targets of CDK5. The results showed that CDK5 could bind to PP2A. Considering our work and the research of Louis et al [20], we speculate that there is a functional correlation between CDK5 and PP2A. However, no relevant studies have revealed the prognostic role of CDK5 and PP2A in gastric cancer.

In this study, immunohistochemistry was used to analyze the correlation between the expression of CDK5 and PP2A and the clinicopathological data in 124 patients with gastric cancer. It was found that the
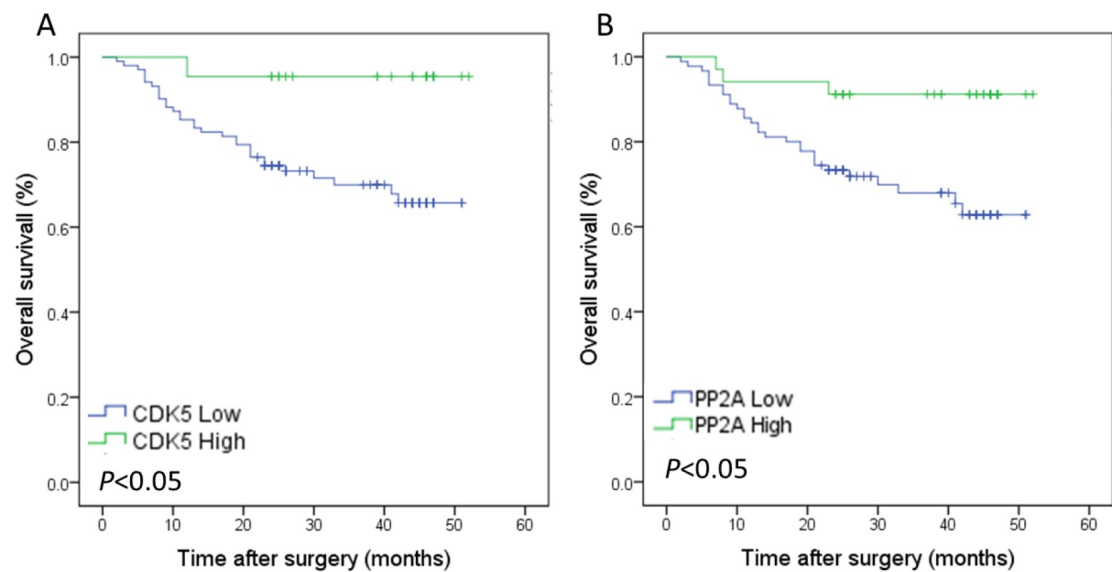

Figure 3. Kaplan-Meier analysis of the correlation between the expression of CDK5 and PP2A and the overall survival of gastric cancer patients. (A) Patients with low expression of CDK5 were compared with the high expression of CDK5.(B) Patients with low expression of PP2A were compared with the high expression of PP2A.
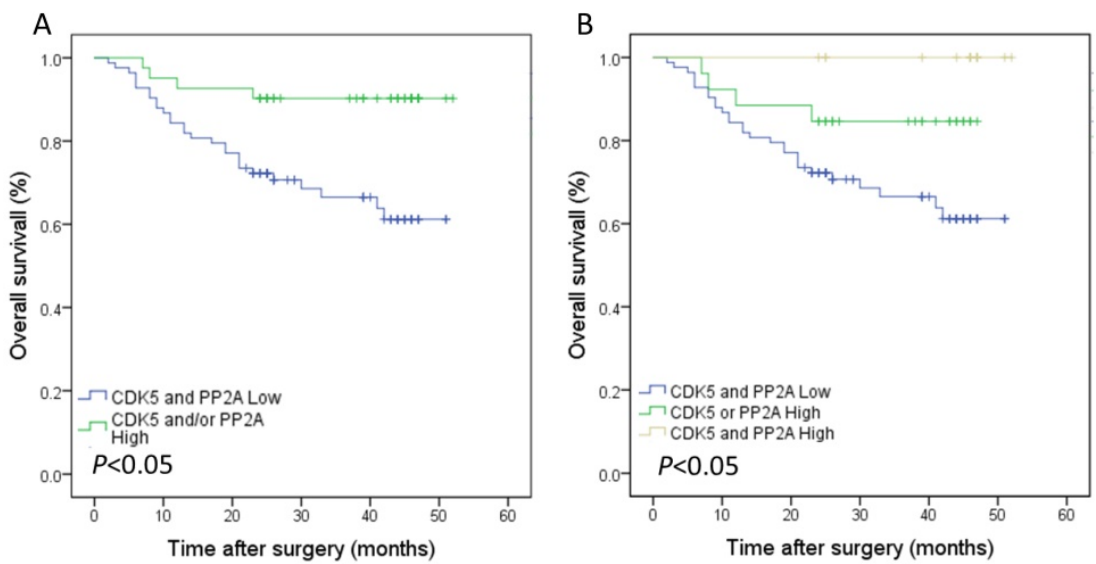

Figure 4. Kaplan-Meier analysis of the correlation between the co-expression of CDK5 and PP2A and the overall survival of gastric cancer patients.(A) Patients with low expression of both CDK5 and PP2A were compared with the rest of the patients.(B) Patients with CDK5 high and/or PP2A high expression were subject to analysis. expression of CDK5 and PP2A in tumor tissues was correlated with TNM stage. A multivariate Cox regression analysis showed that TNM stage and co-expression of CDK5 and PP2A were independent prognostic factors in gastric cancer. CDK5 is an atypical $\mathrm{CDK}$, and recent studies have suggested that CDK5 is involved in the occurrence and development of many types of malignant tumors. CDK5 also plays an important role in many cancers, such as breast cancer, lung cancer, prostate cancer, multiple myeloma and pancreatic cancer. Our study found that the 3-year overall survival rate of gastric cancer patients with low expression of CDK5 was lower than that of patients with high expression of CDK5, and the difference was statistically significant $(P<0.05)$. Most patients with stage I and II disease had tumors with low expression of CDK5, while most patients with stage III and stage IV disease had tumors with high expression of CDK5. It is suggested that CDK5 may be a tumor suppressor in gastric cancer, which is consistent with our previous studies.

Several studies have shown that PP2A is a tumor suppressor: PP2A inhibits tumor genesis via down- regulation of the AKT/TSC1/RAIB/ mTOR pathway, the inactivation of c-Myc, or by antagonizing the $\mathrm{Wnt} / \beta$ -catenin signaling pathway [24]. PP2A can stabilize p53 or cd25 and can thus inhibit tumor growth/ proliferation or induce tumor cell apoptosis [25]. In this study, the prognosis of gastric cancer patients with low expression of PP2A was significantly lower than that of patients with high expression of PP2A. The difference was statistically significant. The majority of patients with stage I and stage II disease had tumors with low expression of PP2A, while those with stage III and stage IV disease had tumors with high expression of PP2A, which suggests that PP2A might play a role in the suppression of gastric cancer.

In addition, we also found a positive correlation between the expression of CDK5 and PP2A in gastric cancer. Among the gastric cancer tissues with high expression CDK5, the expression level of PP2A was higher compared with that 
when CDK5 expression was low. The difference was statistically significant. Moreover, the prognosis of high PP2A/low CDK5 and high CDK5/ low PP2A group was worse than that of both CDK5 and PP2A high expression group, but better than that of both CDK5 and PP2A low expression group. There may be partly because of that the low expression of CDK5 correlation with poor prognostic factors (lymph node metastasis and TNM stage) and low expression of PP2A correlation with (degree of differentiation, lymph node metastasis and TNM stage). Further analysis revealed significant differences in $\mathrm{N}$ stage and TNM stage between patients with low expression of CDK5 and PP2A and other patients. In addition, Louis et al.[20] found that mice knocked out of the PP2A subunit (Ppp2r5d) were able to decrease the phosphatase activity of CDK5 and increase the activity of GSK-3 $\beta$. Therefore, we hypothesized that their (CDK5 and PP2A) impact on prognosis may be related to the overlapping of the two tumor suppressing effects. The accuracy of the combined expression of CDK5 and PP2A in the prediction of the prognosis was better than that of CDK5 or PP2A expression alone, which indicates that the combined expression of CDK5 and PP2A had a better predictive effect on the overall survival rate of gastric cancer patients. More attention should be paid to the role of CDK5 and PP2A in gastric cancer, which requires further study.

There are still some limitations of our study. Fist, only 15 patients $(11.1 \%)$ had both CDK5 and PP2A high expression, therefore, our results need to be validated in a more large-scale study. Second, we evaluated these two tumor makers only by immunohistochemical staining, and focus on the effect of expression patterns of CDK5 and PP2A on the clinicopathological characteristics and the prognosis of patients with gastric cancer. Further study for more information of the possible mechanisms for this finding is necessary in the future.

In summary, low expression of CDK5 and PP2A is closely related to poor prognosis of gastric cancer, and the expression of CDK5 and PP2A is positively correlated in gastric cancer. The co-expression of CDK5 and PP2A is an independent prognostic factor for the overall survival of patients with gastric cancer.
Table 3. Univariate analysis of the correlation between clinicopathological parameters and survival of patients with gastric cancer

\begin{tabular}{|c|c|c|c|}
\hline Variables & 3-year OS rate & Log-Rank Test & $\mathrm{p}$ \\
\hline \multicolumn{4}{|l|}{ Gender } \\
\hline Male & 74.6 & 0.027 & 0.869 \\
\hline Female & 74.1 & & \\
\hline \multicolumn{4}{|l|}{ Age (years) } \\
\hline$>60$ & 76.1 & 0.901 & 0.342 \\
\hline$\leq 60$ & 71.8 & & \\
\hline \multicolumn{4}{|l|}{ Tumor size (cm) } \\
\hline$>5$ & 63.8 & 2.433 & 0.119 \\
\hline$\leq 5$ & 81.4 & & \\
\hline \multicolumn{4}{|l|}{ Tumor location } \\
\hline Lower $1 / 3$ & 77.4 & 3.700 & 0.296 \\
\hline Middle $1 / 3$ & 81.5 & & \\
\hline Upper $1 / 3$ & 62.9 & & \\
\hline More than $1 / 3$ & 91.7 & & \\
\hline \multicolumn{4}{|l|}{ Bormann type } \\
\hline I+ II & 84.0 & 0.896 & 0.344 \\
\hline $\mathrm{III}+\mathrm{IV}$ & 75.6 & & \\
\hline \multicolumn{4}{|l|}{ Degree of differentiation } \\
\hline Well/moderate & 76.3 & 0.609 & 0.435 \\
\hline Poor and not & 73.4 & & \\
\hline \multicolumn{4}{|l|}{ Histological type } \\
\hline Papillary & 68.2 & 4.153 & 0.245 \\
\hline Tubular & 83.2 & & \\
\hline Mucinous & 66.7 & & \\
\hline Signet-ring cell & 81.0 & & \\
\hline \multicolumn{4}{|l|}{ Depth of invasion } \\
\hline $\mathrm{T} 1+\mathrm{T} 2$ & 90.9 & 4.545 & 0.033 \\
\hline $\mathrm{T} 3+\mathrm{T} 4$ & 71.6 & & \\
\hline \multicolumn{4}{|l|}{ Lymph node metastasis } \\
\hline Negative & 94.4 & 4.108 & 0.043 \\
\hline Positive & 71.1 & & \\
\hline \multicolumn{4}{|l|}{ TNM stage } \\
\hline I+II & 97.4 & 14.543 & 0.000 \\
\hline III+IV & 64.2 & & \\
\hline \multicolumn{4}{|l|}{ Vessel invasion } \\
\hline Negative & 79.0 & 1.293 & 0.256 \\
\hline Positive & 72.1 & & \\
\hline \multicolumn{4}{|l|}{ CDK5 expression } \\
\hline Low & 69.9 & 5.964 & 0.015 \\
\hline High & 95.5 & & \\
\hline \multicolumn{4}{|l|}{ PP2A expression } \\
\hline Low & 68.0 & 7.045 & 0.008 \\
\hline High & 91.2 & & \\
\hline \multicolumn{4}{|l|}{ CDK5/PP2A expression } \\
\hline CDK5 and/or PP2A high & 90.2 & 8.122 & 0.004 \\
\hline CDK5 and PP2A low & 66.5 & & \\
\hline
\end{tabular}

Table 4. Multivariate analysis of the correlation between clinicopathological parameters and survival time of patients with gastric cancer

\begin{tabular}{|c|c|c|c|c|c|}
\hline Covariates & Coefficient & Standard Error & HR & 95\% CI for HR & $\mathrm{p}$ \\
\hline $\begin{array}{l}\text { CDK5 and PP2A expression } \\
\text { (low/low vs. high and/or high) }\end{array}$ & -1.075 & 0.544 & 0.341 & $0.118-0.991$ & 0.048 \\
\hline Depth of invasion (T3,T4 vs. T1,T2) & 0.665 & 1.061 & 1.944 & $0.243-15.553$ & 0.531 \\
\hline $\begin{array}{l}\text { Lymph node metastasis } \\
\text { (positive vs. negative) }\end{array}$ & -0.703 & 1.293 & 0.495 & $0.039-6.238$ & 0.587 \\
\hline $\begin{array}{l}\text { Distant metastasis } \\
\text { (positive vs. negative) }\end{array}$ & 0.437 & 0.642 & 1.548 & $0.440-5.446$ & 0.496 \\
\hline TNM stage (stage III + IV vs. I + II) & 2.717 & 1.301 & 15.138 & $1.183-193.739$ & 0.037 \\
\hline
\end{tabular}



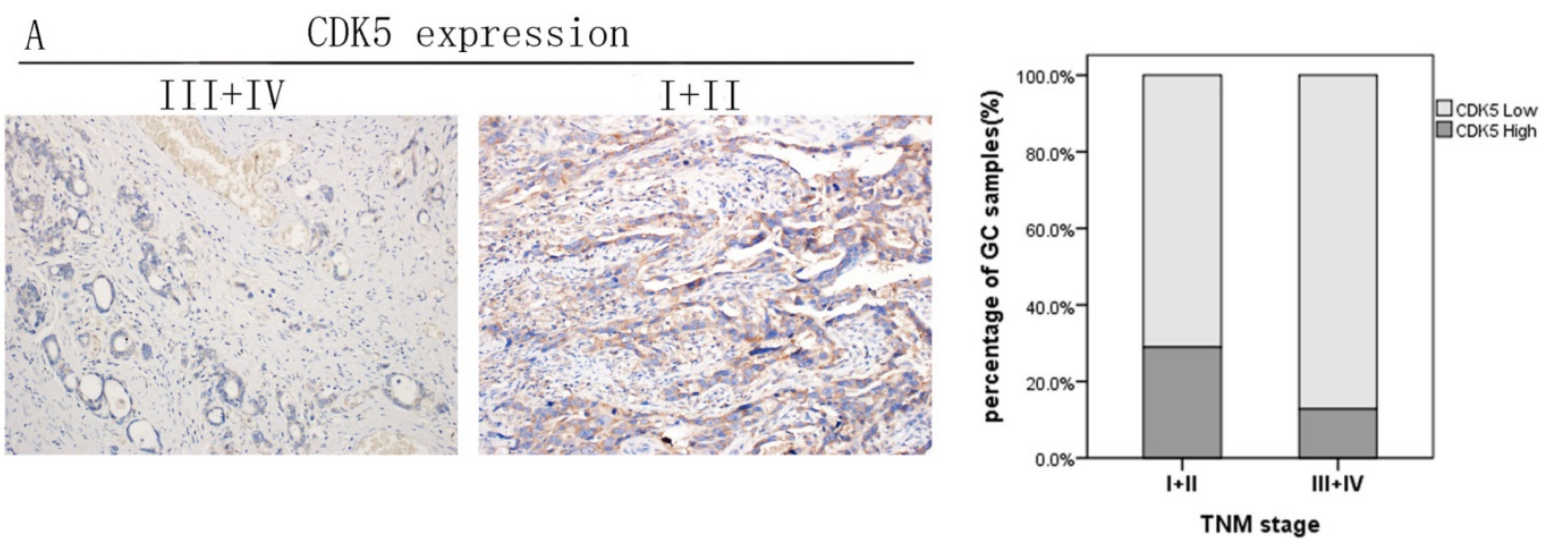

B PP2A expression
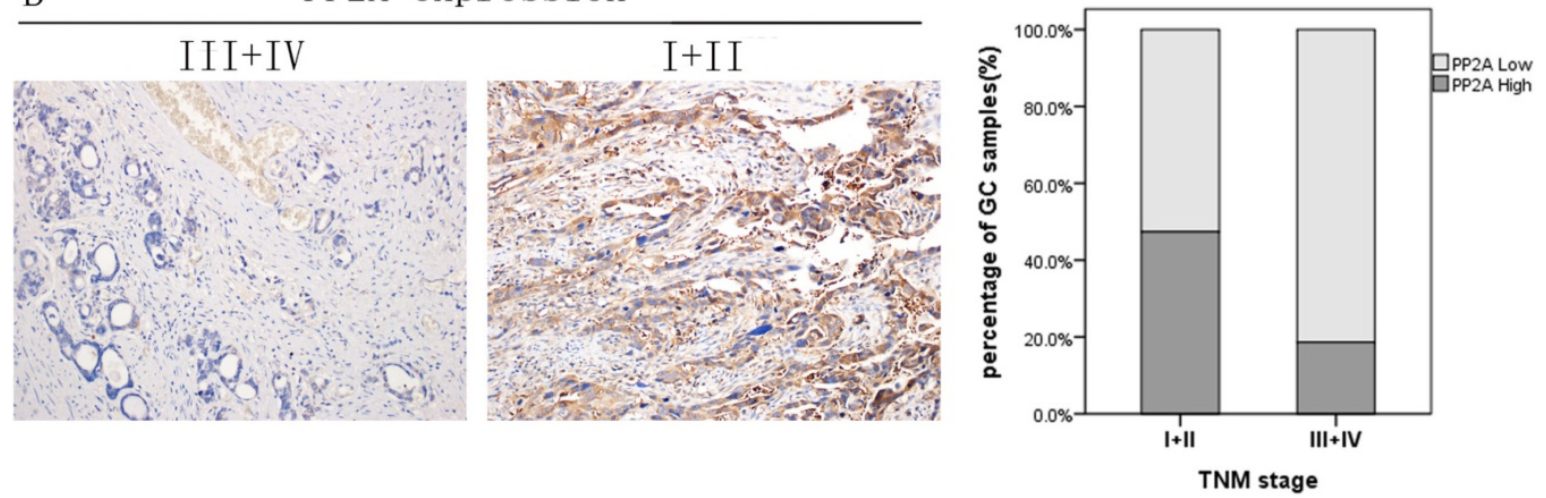

Figure 5. Expression levels of CDK5 and PP2A in gastric cancer tissues with different TNM stage. (A) Negative association of CDK5 expression with gastric cancer TNM stage. (B) Negative association of PP2A expression with gastric cancer TNM stage.
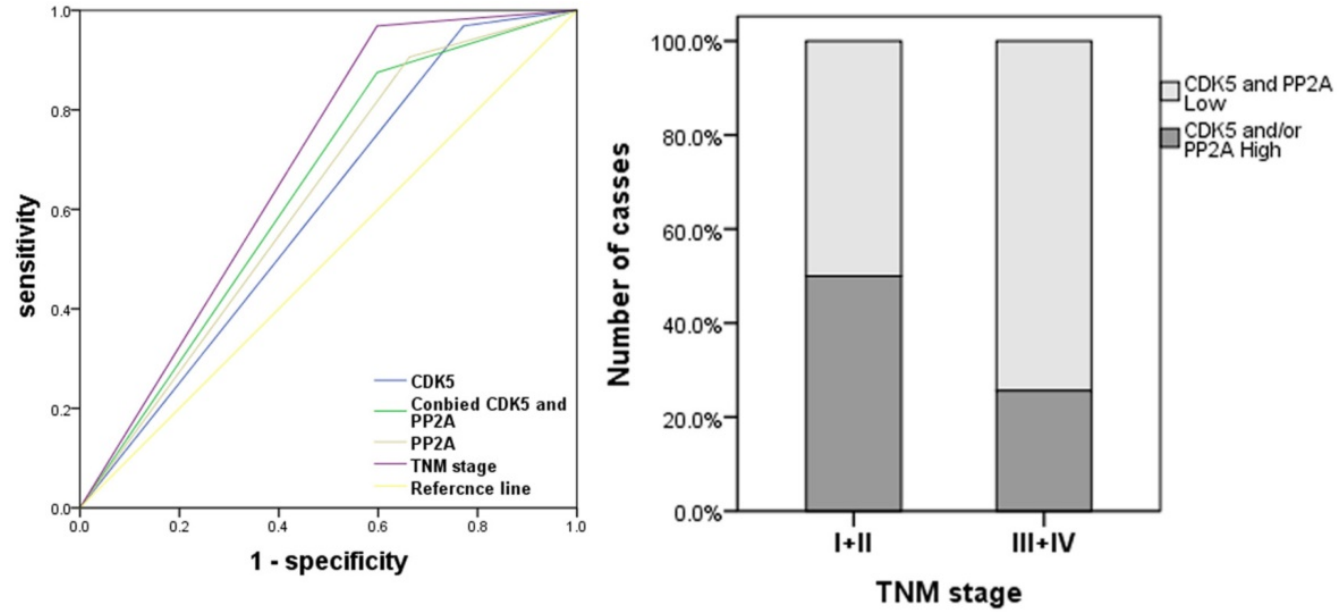

Figure 6. Receiver operating characteristic analysis of the sensitivity and specificity of the predictive value of PP2A expression model, CDK5 expression model, the combined of PP2A and CDK5 model and the TNM model. A: Co-expression of PP2A and CDK5 were significant correlated with TNM stage. B: The area under the ROC curve was $0.639(0.534-0.743)$ for the co-expression of PP2A and CDK5 model, 0.599 (0.493-0.704) for the CDK5 expression model, $0.622(0.517-0.726)$ for the PP2A expression model, 0.685 (0.591-0.780) for the TNM model.

\section{Acknowledgments}

This work was supported by the Science Foundation of the Fujian Province, China (Grant No.2018J01307), Startup Fund for scientific research, Fujian Medical University (No.2016QH024), Scientific and technological innovation joint capital projects of Fujian Province (2016Y9031), Minimally invasive medical center of Fujian Province (2011708\#), Natural Science Foundation of Fujian Province (2016J01463) .

\section{Competing Interests}

The authors have declared that no competing interest exists. 


\section{References}

1. Msph LAT, Bray F, Siegel RL, Jacques Ferlay ME, Lortet-Tieulent J, PhD AJD. Global cancer statistics, 2012. Ca A Cancer Journal for Clinicians. 2015; 65: 69-90.

2. Chen $W$, Zheng R, Baade PD, Zhang S, Zeng H, Bray F, et al. Cancer statistics in China, 2015. Ca A Cancer Journal for Clinicians. 2016; 66: 115.

3. Cruz JC, Tsai LH. A Jekyll and Hyde kinase: roles for Cdk5 in brain development and disease. Current Opinion in Neurobiology. 2004; 14: 390-4.

4. Hisanaga S, Endo R. Regulation and role of cyclin-dependent kinase activity in neuronal survival and death. Journal of Neurochemistry. 2010; 115: 1309.

5. Dempsey LA. Kinase Cdk5 in tumors. Nature Immunology. 2016; 17: 1015.

6. Pozo K, Castro-Rivera E, Tan C, Plattner F, Schwach G, Siegl V, et al. The Role of Cdk5 in Neuroendocrine Thyroid Cancer. Cancer Cell. 2013; 24: 499-511.

7. Henriette M, Zhang S, Thorsten L, Christoph M, Melanie U, Bibb JA, et al. Inhibition of endothelial Cdk5 reduces tumor growth by promoting non-productive angiogenesis. Oncotarget. 2016; 7: 6088-104.

8. Eggers JP, Grandgenett PM, Collisson EC, Lewallen ME, Tremayne J, Singh $\mathrm{PK}$, et al. Cyclin-dependent kinase 5 is amplified and overexpressed in pancreatic cancer and activated by mutant K-Ras. Clinical Cancer Research An Official Journal of the American Association for Cancer Research. 2011; 17: 6140-50.

9. Ehrlich SM, Liebl J, Ardelt MA, Lehr T, De Toni EN, Mayr D, et al. Targeting cyclin dependent kinase 5 in hepatocellular carcinoma - a novel therapeutic approach. Journal of Hepatology. 2015; 63: 102-13.

10. Cao L, Zhou J, Zhang J, Wu S, Yang X, Zhao X, et al. Cyclin-dependent kinase 5 decreases in gastric cancer and its nuclear accumulation suppresses gastric tumorigenesis. Clinical Cancer Research An Official Journal of the American Association for Cancer Research. 2015; 21: 1419.

11. Cristóbal I, Torrejón B, Madoz-Gúrpide J, Rojo F, García-Foncillas J. PP2A plays a key role in inflammation and cancer through tristetraprolin activation. Annals of the Rheumatic Diseases. 2017; 76: annrheumdis-2016-210684.

12. Junttila MR, Puustinen $P$, Niemelão $M$, Ahola $R$, Arnold $H$, Bã Ttzauw T, et al. CIP2A inhibits PP2A in human malignancies. Cell. 2007; 130: 51-62.

13. Pereira G, Schiebel E. Mitotic exit: Determining the PP2A dephosphorylation program. Journal of Cell Biology. 2016; 214: 499.

14. Baldacchino S, Saliba C, Petroni V, Fenech AG, Borg N, Grech G. Deregulation of the phosphatase, PP2A is a common event in breast cancer, predicting sensitivity to FTY720. Epma Journal. 2014; 5: 3.

15. Cristóbal I, Manso R, Rincón R, Caramés C, Senin C, Borrero A, et al. PP2A inhibition is a common event in colorectal cancer and its restoration using FTY720 shows promising therapeutic potential. Molecular Cancer Therapeutics. 2014; 13: 938.

16. Palanichamy K, Kanji S, Gordon N, Thirumoorthy K, Jacob JR, Litzenberg KT, et al. NNMT Silencing Activates Tumor Suppressor PP2A, Inactivates Oncogenic STKs, and Inhibits Tumor Forming Ability. Clinical Cancer Research An Official Journal of the American Association for Cancer Research. 2017; 23: 2325.

17. Zhou X, Updegraff BL, Guo Y, Peyton M, Girard L, Larsen JE, et al. PROTOCADHERIN 7 Acts through SET and PP2A to Potentiate MAPK Signaling by EGFR and KRAS during Lung Tumorigenesis. Cancer Research. 2017; 77: 187-97.

18. Cunningham CE, Li S, Vizeacoumar FS, Lee JS, Parameswaran S, Furber L, et al. Therapeutic relevance of the protein phosphatase $2 \mathrm{~A}$ in cancer. Oncotarget. 2016; 7: 61544

19. Sangodkar J, Farrington CC, Mcclinch K, Galsky MD, Kastrinsky DB, Narla G. All roads lead to PP2A: exploiting the therapeutic potential of this phosphatase. Febs Journal. 2016; 283: 1004.

20. Louis JV, Martens E, Borghgraef P, Lambrecht C, Sents W, Longin S, et al. Mice lacking phosphatase PP2A subunit PR61/B' (Ppp2r5d) develop spatially restricted tauopathy by deregulation of CDK5 and GSK3. Proceedings of the National Academy of Sciences of the United States of America. 2011; 108: 6957-62.

21. Dhavan R, Tsai LH. A decade of CDK5. Nature Reviews Molecular Cell Biology. 2001; 2: 749-59.

22. Kawauchi T, Chihama K, Nabeshima Y, Hoshino M. Cdk5 phosphorylates and stabilizes p27kip1 contributing to actin organization and cortical neuronal migration. Nature Cell Biology. 2006; 8: 17-26.

23. Tsai LH, Delalle I, Caviness VS, Chae T, Harlow E. p35 is a neural-specific regulatory subunit of cyclin-dependent kinase 5 . Nature. 1994; 371: 419-23.

24. Janssens V, Goris J. Protein phosphatase 2A: a highly regulated family of serine/threonine phosphatases implicated in cell growth and signalling. Biochemical Journal. 2001; 353: 417-39.

25. Mi J, Bolesta E, Brautigan DL, Larner JM. PP2A regulates ionizing radiation-induced apoptosis through Ser46 phosphorylation of p53. Molecular Cancer Therapeutics. 2009; 8: 135-40. 